\title{
ANALYSIS OF A SYMMETRIC TRIPLET AND ITS APPLICATION TO RING INSERTIONS
}

\author{
T. d'Amico, G. Guignard, CERN, 1211 Geneva 23, Switzerland
}

\begin{abstract}
It is interesting to study the matching capability of a triplet for a large range of parameters and to cover all the possible solutions. Fully analytical treatments can be used for this purpose, when working with the thin lens approximation. Closed solutions and the conditions in which they exist are described for the special case of triplets with a symmetric geometrical arrangement. They define all possible matchings, which correspond to the requests or limits on the Twiss parameters that are specified, and then define the range of values that are obtainable for the betatron functions. Numerical extension to thick lenses gives the complete solutions for the retained cases. Applications are presented on insertion and lattice problems, with emphasis on the design for a possible isochronous ring that is part of the injection chain of the CLIC drive beam.
\end{abstract}

\section{INTRODUCTION}

The design of a new type of isochronous ring for the injection chain of the CLIC drive beam [1] needs a matching section between two adjacent isochronous cells. The purpose of this section is twofold. First the betatron function at the entrance and at the exit of it should have the same value and its slope should be equal and opposite. Second the total phase advance including the isochronous cell should be $n \pi / m$ where $n$ and $m$ are small integers, in order to minimize the geometrical aberrations. The symmetry of the betatron function leads quite naturally to choose a symmetric lattice for the matching section. We selected a symmetric triplet for its compactness and because it provides the required degrees of freedom. Several authors [2-7] have obtained analytical solutions in the thin-lens approximation for different configurations of quadrupole multiplets which are more general than a simple symmetric triplet. Their number of free variables is different from the six degrees of freedom of the transfer matrix and implies extra constraints or a mismatch factor [8]. In addition the adaptation of the analytical development of the general triplet to the symmetric case is cumbersome and does not take advantage of the lattice symmetry in an easy way.

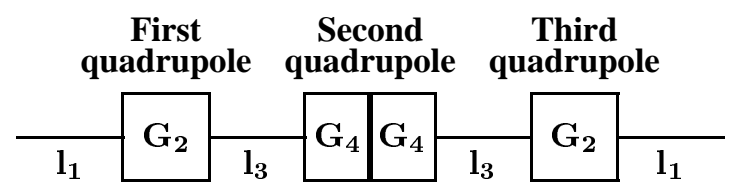

Figure 1: Schematic of a symmetric triplet
A geometrically symmetric triplet as shown in Fig.1 has four free variables (the two drift lengths $l_{1}, l_{3}$ and the magnetic gradients $G_{2}, G_{4}$ respectively of the first and half of the second quadrupole). It can easily be proved [9] that the diagonal elements of the transfer matrix for each plane are equal. Thus the number of independent values which can be fixed by selecting the Twiss parameters at the entrance and at the exit of the triplet is also four. The number of solutions is finite and does not depend upon additional assumptions. This observation has convinced us that a specific study would be useful not only for the design of an isochronous ring but also to explore, in an exaustive way, all the existing solutions for a more general matching problem. A particularly interesting one is related to the experimental insertions of collider rings such as the LHC. Triplets are indeed conveniently used to match the double waist at the interaction point to the rest of the lattice on either side.

\section{COMPATIBLE BETATRON FUNCTIONS FOR A SYMMETRIC TRIPLET}

The equality of the diagonal elements of the transfer matrix for each plane forces an important constraint on the betatron function in that plane at the entrance and at the exit of a symmetric triplet. Neglecting the special case when the phase advance is an odd multiple of $\pi$, we may distinguish two situations. The first one which can be called a mirror symmetry triplet occurs when the betatron function has equal values and its derivative has opposite values, the phase advance being a free parameter. This is the case of interest for building up an isochronous module which may be chained up to form a ring. An example is shown in Fig. 2 . The second configuration occurs when the betatron function has different values at each end of the symmetric triplet with its derivatives being free parameters. This generality is limited by the fact that the phase advance can no longer be freely chosen and is given by :

$$
\cot \mu=\frac{\beta_{2} \alpha_{1}+\beta_{1} \alpha_{2}}{\beta_{1}-\beta_{2}}
$$

where $\alpha_{1}, \beta_{1}$ and $\alpha_{2}, \beta_{2}$ are the Twiss functions at the entrance and exit of the triplet (see Fig. 1) respectively. Thus we have the choice only between two phase advances which differ by $\pi$. The more general case, which will be called a matching triplet can be of interest in several types of insertion. Its application to a collider final focus, where a round beam at the interaction point is matched to the standard periodic FODO cell, has been studied. An example is given in Figs 3 and 4. 


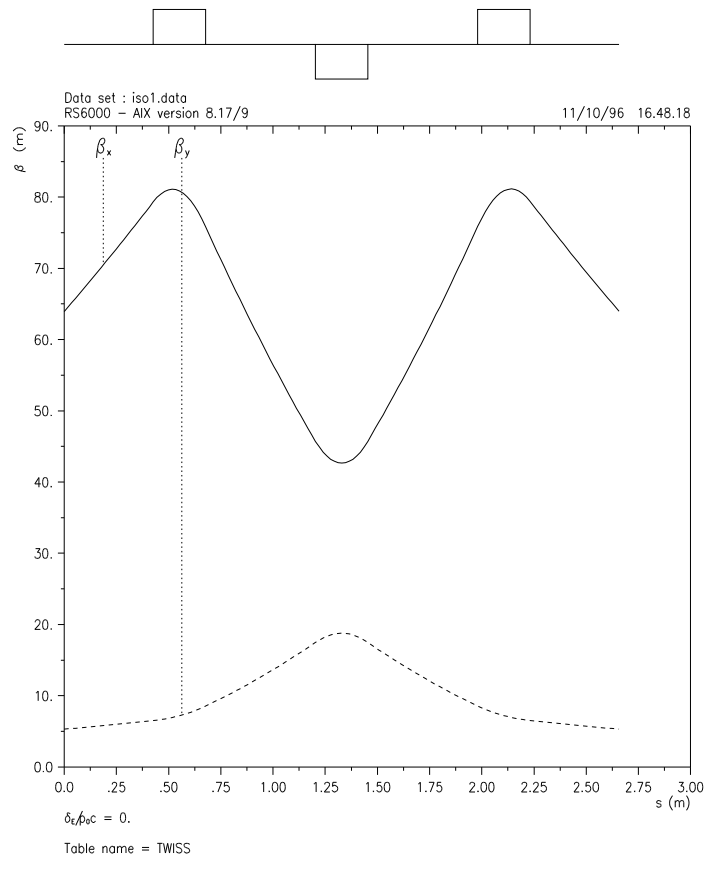

Figure 2: Horizontal and vertical $\beta$-functions of a symmetric triplet which inverts the slopes of these functions.

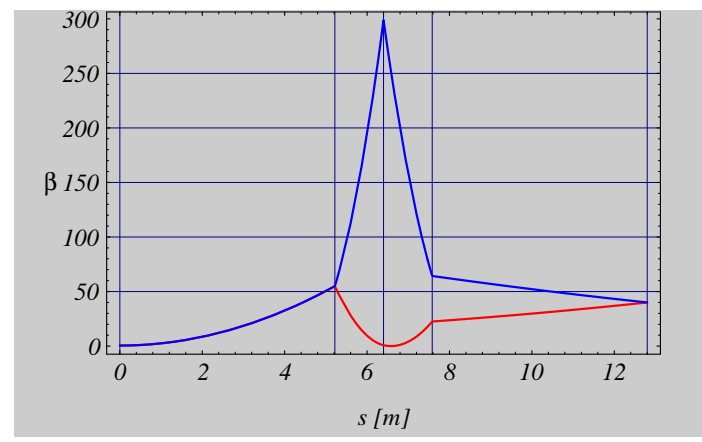

Figure 3: Hor. and vert. $\beta$-functions of the first solution of a symmetric triplet which transforms a round beam with a waist into a round beam with opposite divergences.

\section{DETERMINATION OF THE FOUR PARAMETERS OF A SYMMETRIC TRIPLET}

In the thin lens approximation, the transfer matrices for the horizontal and vertical planes can be expressed as functions of the two drift lengths $l_{1}, l_{3}$ and the quadrupole strengths $g_{2}=K_{2} l_{q}, g_{4}=K_{4} l_{q}$ where $l_{q}$ is the quadrupole length and $K_{2}, K_{4}$ are the normalized gradients. To further simplify the problem the corresponding elements in each plane can be combined by taking half the sum and half the difference of those in the first column (only two elements in each transfer matrix are actually independent). Thus we obtain the following system of equations:

$$
\left\{\begin{array}{l}
c \ell_{1}+\ell_{3}^{2} g_{2} g_{4}=a \\
d \ell_{1}+\ell_{3}\left(g_{2}+g_{4}\right)=b \\
\ell_{3} g_{2}\left(g_{2}+2 g_{4}\right)=c \\
\ell_{3}^{2} g_{2}^{2} g_{4}+g_{2}+g_{4}=d
\end{array}\right.
$$

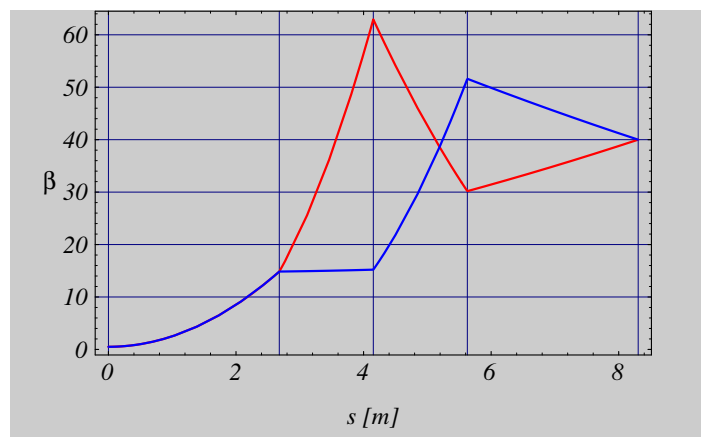

Figure 4: Hor. and vert. $\beta$-functions of the second solution of a symmetric triplet which transforms a round beam with a waist into a round beam with opposite divergences.

where $a, b, c, d$ are defined by:

$$
\begin{gathered}
a=\left(t_{h, 11}+t_{v, 11}-2\right) / 4, \quad b=\left(t_{h, 11}-t_{v, 11}\right) / 4 \\
c=\left(t_{h, 21}+t_{v, 21}\right) / 4, \quad d=\left(t_{h, 21}-t_{v, 21}\right) / 4 .
\end{gathered}
$$

where $t_{h, n m}$ and $t_{v, n m}$ are the elements of the horizontal and vertical transfer matrices respectively. The full analysis of (1) can be found in [9]. Neglecting the very special cases when either $c=0$ or $c= \pm d$ and assuming $b c-a d \neq 0$, the general solution of (1) is given by :

$$
\begin{aligned}
\ell_{1} & =\frac{1}{c^{2}-d^{2}}[(b c-a d) z-(b d-a c)] \\
g_{2} & =\frac{c^{2}-d^{2}}{(b c-a d)\left(1-z^{2}\right)} \\
\ell_{3} & =\frac{(b c-a d)\left(1-z^{2}\right)}{z\left(c^{2}-d^{2}\right)} \\
g_{4} & =\frac{z^{2}(d-c z)}{1-z^{2}}
\end{aligned}
$$

where $z$ is a solution of the cubic equation

$$
z^{3}-\frac{2 d}{c} z^{2}+z+\frac{d^{2}-c^{2}}{c(b c-a d)}=0 .
$$

Of course only real values of $\mathrm{z}$ chosen such that the corresponding drift lengths are positive, should be retained.

\section{APPLICATIONS}

The motivation of the study was to obtain the parameters of the matching triplet between two isochronous modules providing given phase advances in the two planes. The betatron function and its derivative were determined by the isochronous module while the phase advances were chosen in such a way that the total phase advances for the periodic cell were $\pi / 3$ in the horizontal plane and $3 \pi / 2$ in the vertical plane. Thus every three cells in the horizontal plane and two in the vertical plane, kicks due to field errors may cancel out. This is a typical application of mirror symmetry and the results are quite good (Fig. 2). A second interesting 
application is the matching from an interaction point to the standard FODO lattice. This would be schematically the case in circular colliders such as LHC when are neglected the constraints imposed on the value of the first drift length. At the interaction point, the values of the betatron functions in the two planes are equal and relatively small ("low $\beta$ ") while their derivatives are null. At the exit to the triplet it is assumed that the betatron functions in the two planes cross each other with opposite slopes. Thus,

$$
\begin{gathered}
\beta_{h, 1}=\beta_{v, 1}=\beta_{1}, \alpha_{h, 1}=\alpha_{h, 2}=0 \\
\beta_{h, 2}=\beta_{v, 2}=\beta_{2}, \alpha_{h, 2}=-\alpha_{h, 1}=\alpha_{2}
\end{gathered}
$$

The horizontal and vertical transfer matrices are

$$
\begin{aligned}
& T_{h}= \pm 1 / \alpha_{0}\left[\begin{array}{cc}
\alpha_{2} & \beta_{1}-\beta_{2} \\
\gamma_{1}-\gamma_{2} & \alpha_{2}
\end{array}\right] \\
& T_{v}= \pm 1 / \alpha_{0}\left[\begin{array}{cc}
-\alpha_{2} & \beta_{1}-\beta_{2} \\
\gamma_{1}-\gamma_{2} & -\alpha_{2}
\end{array}\right]
\end{aligned}
$$

where $\alpha_{0}=\sqrt{\beta_{1} \gamma_{2}+\beta_{2} \gamma_{1}-2}$ and $\gamma_{1}=1 / \beta_{1}, \quad \gamma_{2}=$ $\left(1+\alpha_{2}^{2}\right) / \beta_{2}$. It is possible to show [11] that two and only two sets of triplet parameters satisfy these transfer matrices if we neglect a difference of $\pi$ in the corresponding phase advances. The only condition is that $\alpha_{2}$ should not be zero or equal to $\sqrt{\left(\beta_{2}-\beta_{1}\right) / \beta_{1}}$. These two sets of parameters are given by:

$$
\text { a) } \begin{aligned}
l_{1} & =\frac{\alpha_{2} z_{r, 1}+\alpha_{0}}{\left|\gamma_{1}-\gamma_{2}\right|} \\
g_{2} & =\frac{\gamma_{1}-\gamma_{2}}{\alpha_{2}} \frac{1}{1-z_{r, 1}^{2}} \\
l_{3} & =2 l_{1} / z_{r, 1}^{2} \\
g_{4} & =\frac{\alpha_{2} z_{r, 1}^{3} g_{2}}{2 \alpha_{0}}
\end{aligned}
$$

where $z_{r, 1}$ is the (only) real solution of the cubic equation $z^{3}+z+2 \alpha_{0} / \alpha_{2}=0$

$$
\begin{gathered}
f=1 / g_{2}=\frac{\alpha_{2}}{\gamma_{1}-\gamma_{2}} \\
l_{1}=\left|f / \alpha_{2}\right| \sqrt{\alpha_{0}\left(\alpha_{0}+\left|\alpha_{2}\right|\right)} \\
l_{3}=|f| \sqrt{1+\left|\alpha_{2} / \alpha_{0}\right|} \\
g_{4}=-g_{2} / 2 .
\end{gathered}
$$

This treatment has been included in the package BeamOptics developed using the Mathematica language [10]. When $l_{1}$ is imposed the approach described here can be extended by adding a drift of free length at the exit of such a symmetric triplet. It is then possible to examine the behaviour of important quantities such as the length of this drift and the maximum value reached by the betatron functions inside the triplet; and this according to the variation of the triplet parameters in a predefined range, and to specific constraints on the betatron functions at the end of the added drift. Two cases have been investigated : waist in one plane and equal values of the betatron function with opposite slopes. All the possible solutions can be determined for canonical triplets $\left(g_{4}=-g_{2}\right)$, general symmetric triplets and even triplets where the third quadrupole strength is slightly modified. This study was applied to insertions of LHC type and provided interesting results on the ranges of acceptable triplet parameters [11].

\section{CONCLUSIONS}

It has been shown that in the thin lens approximation a geometrically symmetric triplet is a structure simple enough to be handled analytically and yet powerful enough to be applied to several interesting and quite different lattices. The method provides in a direct way all the existing solutions permitting a complete study of the required lattice. Existence conditions can only be made explicit in special cases but readily constructed as an abacus whenever needed. It can be shown [9] that the extension to thick lenses is easily obtained by solving a system of two nonlinear equations functions of the normalized gradients $K_{2}, K_{4}$. Of course such results are obtainable also by standard numerical matching programs although they may have difficulties in converging if not conveniently guided. Moreover their use is cumbersome and time consuming when included in a chain of specific codes written in other languages. The thin lens approximation obtained by the method described here is an excellent starting point for such algorithms.

\section{REFERENCES}

[1] T.E. d'Amico, G. Guignard, 'First-order design of a new type of isochronous arc', CERN SL/95-120, 1995.

[2] E. Regenstreif, 'Phase-space transformations by means of quadrupole multiplets', CERN 67-6, 1967.

[3] G. Wüstefeld, R. Maier and B. Simon, 'The analytical lattice approach for the ring design of BESSY II', Proc. First European Part. Accel. Conf. (EPAC), Rome, 1988.

[4] B. Zotter, 'Calculation of the parameters of a quad triplet from the transfer matrices', CERN/ISR-TH/73-43, 1973.

[5] B. Autin, E. Wildner, 'Applications of symbolic techniques to structural beam optics', Proc. Fifth European Part. Accel. Conf. (EPAC), Sitges (Spain), 1996.

[6] O. Napoly, 'Thin lens telescopes for final focus systems', CERN/LEP-TH/89-69, CLIC Note No. 102, 1989.

[7] P.J. Bryant, K. Johnsen,'The principles of circular accelerators and storage rings', (Cambridge University Press, 1993).

[8] P.J. Bryant, private communication.

[9] T. E. d'Amico, 'General treatment of a matching quadrupole triplet which is symmetric around its median plane', CLIC Note 322, January 1997.

[10] B. Autin, T. d'Amico, V. Ducas, M. Martini, E. Wildner, 'Analytic lattice design with BeamOptics' Proceedings of this Conference.

[11] T. E. d'Amico, G. Guignard, 'Constraints in the use of a symmetric triplet for the LHC low $\beta$ insertion ', to be published. 